\title{
Tuning the mechanical behaviour of structural elements by electric fields
}

\author{
Luigi Di Lillo, ${ }^{1, a)}$ Wolfram Raither, ${ }^{1}$ Andrea Bergamini, ${ }^{2}$ Manuel Zündel, ${ }^{1}$ \\ and Paolo Ermanni ${ }^{1}$ \\ ${ }^{1}$ ETH Zurich, Centre of Structure Technologies, Leonhardstrasse 27, 8092 Zurich, Switzerland \\ ${ }^{2}$ Empa, Swiss Federal Laboratories for Materials Testing and Research, 8600 Dubendorf, Switzerland
}

(Received 22 April 2013; accepted 21 May 2013; published online 7 June 2013)

\begin{abstract}
This work reports on the adoption of electric fields to tune the mechanical behaviour of structural elements. A mechanical characterization procedure, consisting of double lap joint and 3-point bending tests, is conducted on copper-polyimide laminates while applying electric fields of varying intensity. Field dependence and, thus, adaptability of shear strength and bending stiffness are shown as a function of the overlapping length and interfaces number, respectively. Further, the impact of remaining charges is investigated in both testing configurations. The findings herein lay the foundation for the implementation of electro-adaptive components in structural applications. (C) 2013 AIP Publishing LLC. [http://dx.doi.org/10.1063/1.4809728]
\end{abstract}

The development of new aerospace and automotive products has been enabled by remarkable progress in lightweight and composite structures disciplines. ${ }^{1,2}$ Despite the adoption of new materials, which have allowed increasing their stiffness and strength to weight ratio, typically their mechanical behaviour is (a) time-invariant and (b) design driven. Specifically, any external load exerted on them is expected to yield the same response at all times. The resulting designs are necessarily sub-optimal tradeoffs, as they are sized for a wide range of operating conditions. These conditions often include very rare and unlikely operational states that are, however, critical and thus constitutive for the design, although compromising the performance during most of the operating time. The appearance of adaptive structures provides new degrees of freedom to designers since adaptivity allows the structure to respond differently to each operating conditions and to perform optimally in each one of them. ${ }^{3}$

In this framework, airfoils having adaptive capabilities, i.e., morphing airfoils, are the object of considerable research efforts in civil and military applications. ${ }^{4-6}$ The benefit of morphing capabilities is also postulated for wind turbine blades of the upcoming generations (with blade lengths exceeding $100 \mathrm{~m}$ ). ${ }^{7}$ Elements with variable bending/torsional stiffness capabilities are in demand for realizing such morphing concepts.

Electro bonded laminates (EBL) are capacitor-like multilayered structures able to switch, on demand and reversibly (off/on-voltage), their capability of shear stress transfer and, consequently, their stiffness components like the bending stiffness $T$. In particular, they can also directly meet the need for elements with adaptive shear strength arising from structural concepts for morphing based on this feature. ${ }^{8}$

The application of a voltage signal on EBL steers charges migration from one conductive plate to the other determining the appearance of charges with opposite sign on two facing electrodes. This capacitor-charging process, in turn, determines the onset of Coulomb attractive forces between the conductive plates. ${ }^{9}$ Molecular adhesion and surface roughness cause resistive forces of friction to take place. ${ }^{10}$ It is postulated,

a)E-mail: dilillo@mavt.ethz.ch; wraither@ethz.ch theoretically, ${ }^{11}$ that the presence of friction combined with the appearance of Coulomb attractive forces lead to the formation of a physically bonded state and allow, by means of voltage control, (a) to adjust the shear strength of the bonding and (b) to tune the bending stiffness of the structure (by controlling the number of interfaces electro-mechanically connected).

The shear stress $\tau_{\perp \|}$ transferable at the interfaces of an EBL is given in Eq. (1). It is dependent on the normal stress $\sigma_{\perp}$ (Maxwell stress ${ }^{12}$ ) that it exhibits under the influence of an applied electric field $E$,

$$
\tau_{\perp \|}=\mu \sigma_{\perp}=\mu \frac{1}{2} \varepsilon_{0} \varepsilon_{\mathrm{r}} E^{2} .
$$

In Eq. (1), $\varepsilon_{0}$ is the vacuum permittivity, $\varepsilon_{\mathrm{r}}$ is the relative permittivity of the dielectric layers constituting the EBL, and $\mu$ is the static coefficient of friction (COF) between the layers.

The relative change in bending stiffness of an EBL consisting of $n$ electrode layers and $(n-1)$ dielectric layers, following voltage application, is (in a purely geometric formulation) expected to be proportional to the number of layers squared. ${ }^{13}$ Considering a difference in electrodes and dielectric thicknesses ( $t_{\mathrm{e}}$ and $t_{\mathrm{d}}$, respectively) and assuming the Young's modulus of the dielectric layers $Y_{\mathrm{d}}$ to be much smaller than the one of the electrodes $Y_{\mathrm{e}}$, a more general expression of the stiffness ratio reads,

$$
\frac{T_{\text {on }}}{T_{\text {off }}}=n^{2} \frac{\left(t_{\mathrm{e}}+t_{\mathrm{d}}\right)^{2}}{t_{\mathrm{e}}^{2}}-\frac{t_{\mathrm{d}}\left(2 t_{\mathrm{e}}+t_{\mathrm{d}}\right)}{t_{\mathrm{e}}^{2}} .
$$

Previous works have implemented, experimentally, EBL in vibration suppression applications. ${ }^{13}$ A detailed electromechanical characterization of EBL, showing their effective shear strength and bending stiffness behaviour as a function of layers' number and voltage intensity, is still missing but greatly needed. The purpose of this work is to focus on these aspects in order to fully exploit the potential of EBLs, define their limitations, and predict their behaviour when introduced in structural applications. This work investigates also the impact of charge injection at the dielectric/electrode interface on preventing the realization of a fully compliant state (0-stiffness) when the voltage is turned off. 
The electro-mechanical characterization of EBL, Fig. 1, has been carried out through double lap shear and 3-point bending tests on polyimide (PI, $25 \mu \mathrm{m}$ ) layers, supplied by $\mathrm{UBE}^{\circledR}$, Germany, properly cut with a Zünd M-1600 cutting machine in rectangular shapes of dimensions $l_{d}=100 \mathrm{~mm}$, $b_{d}=30 \mathrm{~mm}$. The electric signal was supplied through a high voltage amplifier Trek 10/10C. The mechanical forces were exerted by means of a Zwick/Roell Z005 tensile testing machine, endowed with a $100 \mathrm{~N}$ load cell. The conductive plates consisted of laser-cut copper $(\mathrm{Cu}, 50 \mu \mathrm{m})$ layers in dimensions $b_{e}=12.7 \mathrm{~mm}, l_{e}=150 \mathrm{~mm}, t_{e}=50 \mu \mathrm{m}$ with a radius of curvature of $b_{e} / 2$ for the double lap shear tests. As for the 3-point bending measurements, the conductive plates were laser-cut in rectangular shapes of dimensions $d_{0}=80 \mathrm{~mm}(d=60 \mathrm{~mm}), b_{e}=15 \mathrm{~mm}$ and a radius of curvature of $1 \mathrm{~mm}$. Only the contact regions of the facing electrodes were shaped. The shaping aims at limiting the charge accumulation capabilities and, then, the presence of electric fields peaks at the electrodes' edges which would lead to premature electric breakdown events. The values of dielectric strength and relative permittivity that we measured on alike

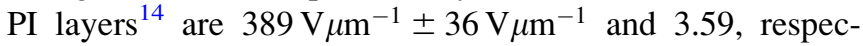
tively. In both testing configurations, a safety factor on the maximum applied voltage bigger than $50 \%$ of the PI dielectric strength was applied.

Fig. 1 reports, schematically (left) and in photos (right), the two characterization methods we set out. The double lap shear configuration shown on top has served to evaluate the capability of EBL to transfer shear stress at the interfaces and to corroborate the theoretical formulation expressed in Eq. (1). In a previous work, ${ }^{15}$ we highlighted the effect of bending moment, investigated by varying EBL electrodes' thickness, on their capability to transfer shear stress when arranged in single lap shear configurations. Double lap shear arrangements, by virtue of their symmetric geometry, have allowed reducing drastically the influence of the bending moment and enabled, therefore, to record unaffected shear strength values. The 3-point bending configuration shown on the bottom permitted to assess the quality and the level of electro-mechanical bonding between the different layers constituting the EBL. The voltage application is, indeed, expected to bring the loose stack of layers in a fully bonded configuration with a stiffness change quantified by Eq. (2).
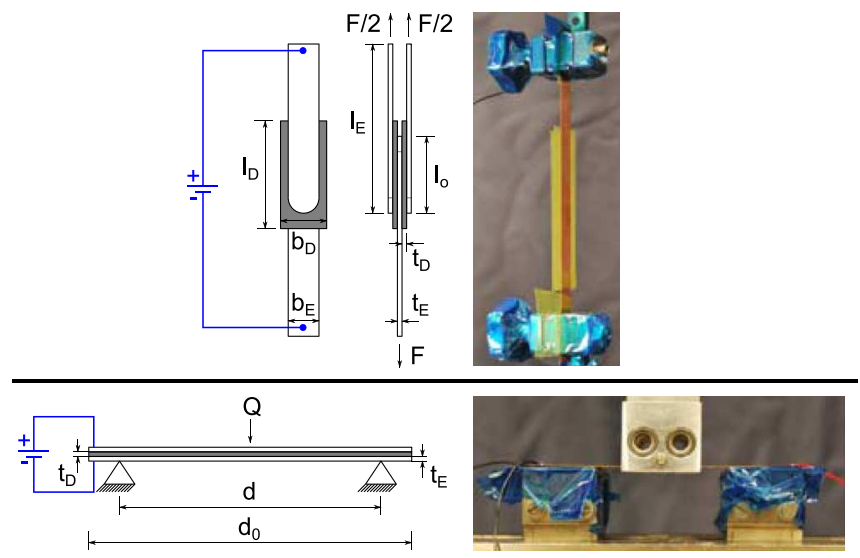

FIG. 1. Drawings (left) and photos (right) of double lap shear (top) and 3-point bending (bottom) configurations.
In both testing configurations, an adhesive tape (blue layers appearing in Fig. 1) is used to insulate the Zwick/Roell tensile testing machine from the conductive plates of the EBL.

In Fig. 2, we report the experimental results of double lap shear tests carried out at different EBL's overlapping lengths, $20 \mathrm{~mm}, 40 \mathrm{~mm}$, and $80 \mathrm{~mm}$. Each point in the graph is the mean value resulting from 5 measurements and the vertical error bars represent the standard deviation about the mean value. On the y-axis, we report the mean transferable shear strength $\tau_{\mathrm{m} \text { max }}$ (averaged over the surface) obtained as a result of the measured applicable pulling force recorded at the onset of sliding (i.e., maximum force $F$, Fig. 1), divided by twice the overlapping area (two interfaces in double lap shear), over different target voltages $U$, x-axis. The graph in Fig. 2 represents, therefore, the maximum shear stress that EBL can transfer at a given voltage and overlapping length which is thus referred to as mean shear strength in this work.

The data points summarized in Fig. 2 are obtained from force-displacement (FD) experimental curves and, for the sake of clarity, we report a typical FD curve in Fig. 3 (in particular, the behaviour recorded at $3500 \mathrm{~V}$ for an overlapping length of $20 \mathrm{~mm}$ is reported). In addition to the mentioned transition from static to dynamic friction, determining the EBL's shear strength, two further distinct regimes can be identified in the experimental data: (a) a non-linear FD behaviour at lower displacements highly likely due to initial straightening of the sample, followed by (b) an almost linear response representing the elastic loading of the EBL. In the latter, the small deviation from ideal linearity can be assumed to result from local dynamic friction at parts of the interfaces.

The analytical curve in Fig. 2 ensues from Eq. (1). The value of the $\mathrm{COF}$ was found to be $\mu=0.28 \pm 0.01$. It has been measured on the same PI film at an effective normal load value of $61.37 N \pm 3.65 N$, by way of the experimental setup detailed by Gines et al. ${ }^{16}$ Analytical considerations predict a hyperbolic shear stress distribution along the length of an overlap loaded by pure shear ${ }^{17}$ showing maxima at the edges whose influence increases with increasing overlapping length. However, there is a noticeably good agreement

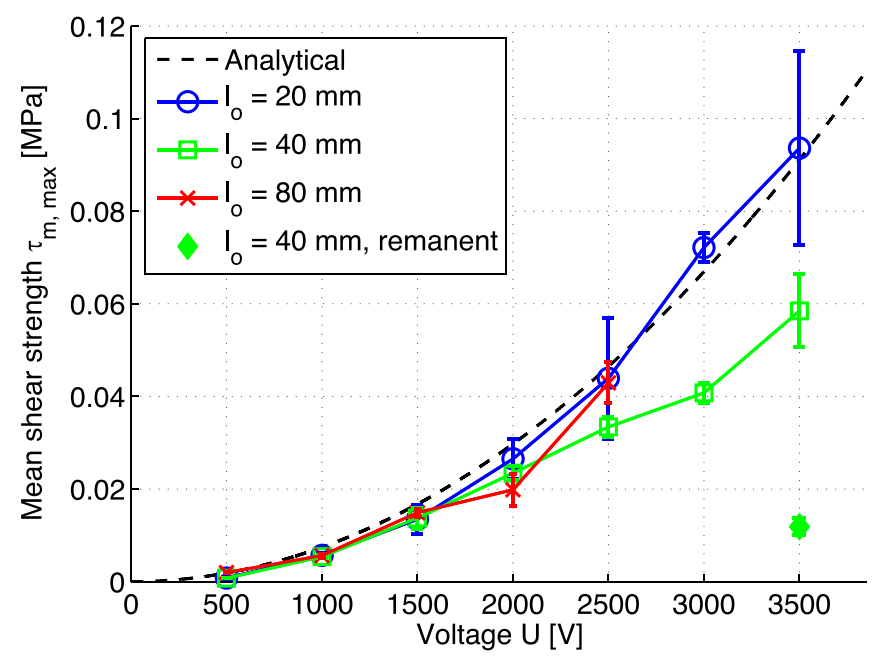

FIG. 2. Mean shear strength at different target voltages for different EBL overlapping lengths $l_{o}$. Analytical curve in black dashes from Eq. (1) with $Y_{\mathrm{e}}=120 \mathrm{GPa}$ and $Y_{\mathrm{d}}=3 \mathrm{GPa}$. 


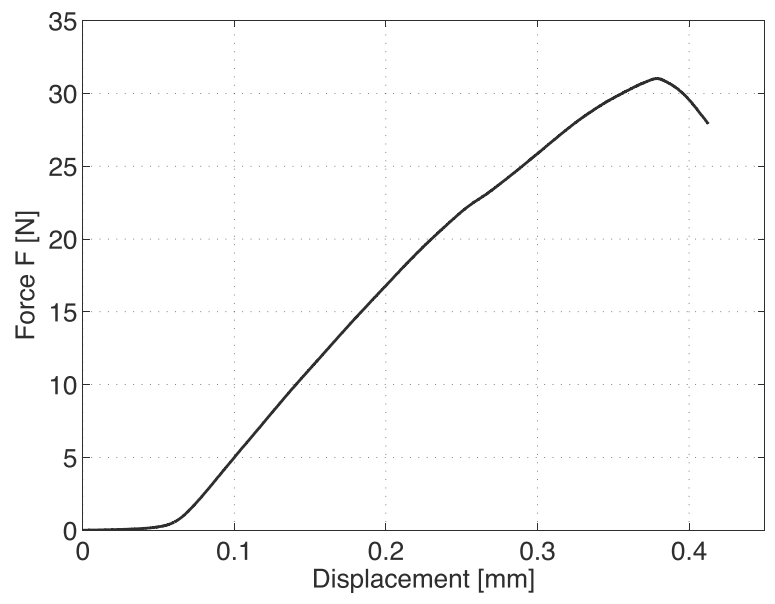

FIG. 3. Force-displacement behaviour of an EBL arranged in a double lap shear configuration supplied with $3500 \mathrm{~V}$.

between the experimental results and the behaviour expected by considering only the mean shear stress. This is particularly true for the shortest overlapping length and up to a certain voltage for the intermediate overlapping length. It can thus be concluded that the influence of shear stress gradients on the shear strength as defined here is limited under these conditions. The target voltages were chosen in relation to the overlapping length: longer overlaps would statistically be more prone to breakdown events due to larger chances of having defects, impurities, dust particles. ${ }^{18}$ Indeed, despite an expected dielectric strength of $389 \mathrm{~V} \mu \mathrm{m}^{-1}$, we recorded breakdown phenomena at fields of $100 \mathrm{~V} \mu \mathrm{m}^{-1}$ for $80 \mathrm{~mm}$ overlaps. In addition to this effect, the limitations set by the maximum load of the load cell $(100 \mathrm{~N})$ and yield point of the electrodes, constrained us from applying voltages larger than $2500 \mathrm{~V}$ on this overlapping pattern. The $40 \mathrm{~mm}$ was capable of standing electric fields as high as those obtained across the 20 mm overlap.

Another aspect we investigated concerned the presence of remaining charges in the dielectric layers due to charge injection ${ }^{19}$ that might take place at high electric fields and affect the behaviour of the EBL after the voltage has been turned off. Indeed, in the off-state, the EBL stiffness components should be zero but the presence of remaining charges could compromise this feature. Thereby, measurements of remaining force in the same double lap shear configurations have been performed to investigate and quantify this aspect. In Fig. 2, the resulting remaining mean shear strength (average value from 5 samples and the resulting standard deviation) is reported as well. The measurements consisted in applying a voltage of $3500 \mathrm{~V}$ (highest reachable prior to breakdown) on the $40 \mathrm{~mm}$ overlapping samples for $60 \mathrm{~s}$ and, right after, starting the pulling phase without any voltage applied. As shown in Fig. 2, a mean shear strength different from zero ( $25 \%$ of the instance with applied voltage) was recorded. Consequently, it is highly unlikely to take the system back to a 0 -stiffness state when the voltage is removed. This aspect will have a relevance on the EBL's implementation in structures and will call for possible dielectric layers' surface treatments. ${ }^{20}$

The change in bending stiffness of EBLs composed by $\mathrm{Cu} / \mathrm{PI}$ as constituent materials and geometry defined in Fig. 1 has been studied and evaluated through 3-point bending tests, Fig. 4. In Fig. 4, positive quarter, the experimental results about two different arrangements, 2 and $4 \mathrm{Cu} / \mathrm{PI}$ interfaces, left and right plots, respectively, are reported. Each plot shows in continuous lines the measured force over maximum deflection $w$ of each arrangement at 3 different target voltages $(0 \mathrm{~V}, 2000 \mathrm{~V}$, and $4000 \mathrm{~V})$ together with the theoretically decoupled (off-voltage) and bonded (on-voltage) curves in dashed lines. The theoretical bonded state is the configuration obtained when considering the material perfectly bonded, with no interfaces. It was expected that (a) any small voltage, no matter the intensity, would have led to a perfectly electro-mechanically bonded state and that (b) the quality of the bonding (i.e., how well the arrangement resembles the bonded state) was irrespective of the number of interfaces since the presence of the electrical signal (voltage in this work) would affect only the shear strength of the EBL. Yet, it can be concluded from a comparison of the slopes of the curves that, in both cases, (a) for a certain force $Q$ and a certain deflection $w$, a given minimum voltage (i.e., not any voltage different from zero) is required to bring the system to a bonded configuration and that (b) this specific voltage depends on the number of interfaces. Particularly, while a voltage signal of $4000 \mathrm{~V}$ is sufficient to bring the EBL with two interfaces to a bonded state from the beginning (0 deflection), in the case of 4 interfaces at the same voltage the bonded configuration is achieved only in a certain deflection range (with the lower margin bigger than 0) due to higher likelihood of pronounced initial sliding with increasing number of interfaces. Another aspect to emphasize is the absence of a purely linear regime for defining the bending stiffness of the EBL. We are, in fact, not providing the results of the bending stiffness for the two configurations investigated since its values should be extrapolated from the
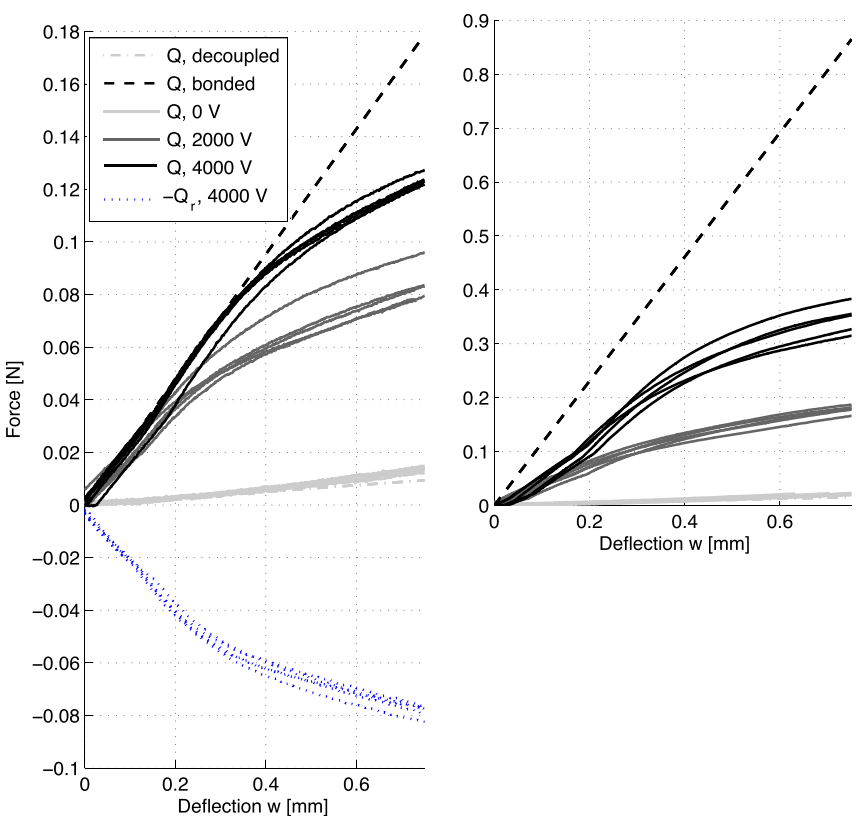

FIG. 4. Force $Q$ versus deflection behaviour of EBL (2 interfaces, left, and 4 interfaces, right) in 3-point bending for different voltages. Bonded and decoupled ideal curves based on Eq. (2). Remanent force $Q_{\mathrm{r}}$ in the negative quarter. 
experimental curves and, as the graphs in Fig. 4 clearly show, they cannot be univocally defined for the whole range of deflections. However, for the 2-interfaced EBL, the good fit between experimental and theoretical curves up to a certain deflection leads us to conclude that, from a certain voltage $(2000 \mathrm{~V})$ and in a given deflection range, Eq. (2) is fulfilled.

We investigated the role of the remaining charges on 2-interfaced EBLs also in the 3-point bending arrangements. Particularly, the negative quarter of Fig. 4 presents, in dashed blue lines, the experimental results of the remaining force $Q_{\mathrm{r}}$ as a function of deflection $w$ of 5 EBLs. The curves have been obtained following the same procedure we adopted for the double lap shear tests: voltage application $(4000 \mathrm{~V}$ in this case) for $60 s$ and deflections measurement afterwards with no voltage applied. We reported the results in the negative quarter for sake of clarity (note the sign $-Q_{\mathrm{r}}$ in the top left inset of the figure). The recorded values of remaining force versus deflection are as much as $65 \%$ with respect to the case with applied voltage. The impact of charge injection processes is much more relevant in the EBLs bending component than in their shear stress transfer capabilities.

In this work, an experimental study on the mechanical response of $\mathrm{Cu} / \mathrm{PI}$ EBLs has been carried out. The results of the analyses have been compared with the theoretical formulations. A good agreement has been found for some overlapping lengths of double lap shear configurations and, starting from a certain voltage, for the 2-interfaced EBLs in 3-point bending tests. The role of the remaining charges has been quantified at the highest allowable (in terms of breakdown voltage) voltage and for particular double lap shear and 3 -point bending configurations. The results show that their effect is more pronounced in the latter case. With a view to EBL application in morphing structures, methodologies to reduce their contribution have to be figured out. Moreover, better dielectric layers have to be adopted to improve the mechanical quantities (shear strength, bending stiffness) of interest. To this purpose, multilayer dielectric films ${ }^{21}$ have been identified as suitable candidates. In closing, cyclic electromechanical tests will be performed for assessing the reliability, the durability, and the fatigue behaviour of EBL's interfaces.

L. Di Lillo, W. Raither, and A. Bergamini contributed equally to the scientific results and to the redaction of this article. The authors wish to thank the Swiss National Science Foundation for project funding as part of the NRP 62. Rebekka Ginés from the Structural Engineering Laboratory of EMPA is acknowledged for friction tests.

${ }^{1}$ J. Immarigeon, R. Holt, A. Koul, L. Zhao, W. Wallace, and J. Beddoes, Mater. Charact. 35, 41 (1995).

${ }^{2}$ A. K. Noor, "Structures technology: historical perspective and evolution," AIAA Paper No. 132, 1998.

${ }^{3}$ A. K. Noor, S. L. Venneri, D. B. Paul, and M. A. Hopkins, Comput. Struct. 74, 507 (2000).

${ }^{4}$ A. Sofla, S. Meguid, K. Tan, and W. Yeo, Mater. Des. 31, 1284 (2010).

${ }^{5}$ T. A. Weisshaar, "Morphing aircraft technology - New shapes for aircraft design,” Tech. Report RTO-MP-AVT-141, DTIC Document, 2006.

${ }^{6}$ A. K. Jha and J. N. Kudva, Proc. SPIE 5388, 213 (2004).

${ }^{7}$ J. Van Wingerden, A. Hulskamp, T. Barlas, B. Marrant, G. Van Kuik, D.-P. Molenaar, and M. Verhaegen, Wind Energy 11, 265 (2008).

${ }^{8}$ W. Raither, A. Bergamini, and P. Ermanni, J. Intell. Mater. Syst. Struct. 24, 334 (2013).

${ }^{9} \mathrm{C}$. A. Coulomb, Premier-[troisième] mémoire sur l'electricité et le magnétisme (Académie Royale des sciences, 1785), pp. 569-577.

${ }^{10}$ I. M. Hutchings, Tribology: Friction and Wear of Engineering Materials (Butterworth-Heinemann Ltd., Oxford, 1992).

${ }^{11}$ A. Bergamini, R. Christen, B. Maag, and M. Motavalli, Smart Mater. Struct. 15, 678 (2006).

${ }^{12}$ J. C. Maxwell, A Treatise on Electricity and Magnetism (Clarendon Press, Oxford, 1881), Vol. 1.

${ }^{13}$ A. Bergamini, R. Christen, and M. Motavalli, Smart Mater. Struct. 16, 575 (2007).

${ }^{14}$ L. Di Lillo, D. Carnelli, A. Bergamini, S. Busato, and P. Ermanni, Smart Mater. Struct. 20, 057002 (2011).

${ }^{15}$ L. Di Lillo, W. Raither, C. Di Fratta, A. Bergamini, and P. Ermanni, in Proceedings of American Society Mechanical Engineers, SMASIS (2012).

${ }^{16}$ R. Gines, A. Bergamini, R. Christen, M. Motavalli, and P. Ermanni, "Frictional behaviour of polymer films under mechanical and electrostatic loads," Smart Mater. Struct. (submitted).

${ }^{17}$ W. Hovgaard, Proc. Natl. Acad. Sci. U.S.A. 16, 673 (1930).

${ }^{18} \mathrm{M}$. Chanda and S. Roy, Plastics Technology Handbook (CRC, New York, 2006).

${ }^{19}$ M. Ieda, IEEE Trans. Electr. Insul. EI-22, 261 (1987).

${ }^{20}$ Z. An, Q. Yang, C. Xie, Y. Jiang, F. Zheng, and Y. Zhang, J. Appl. Phys. 105, 064102 (2009).

${ }^{21}$ M. Mackey, A. Hiltner, E. Baer, L. Flandin, M. A. Wolak, and J. S. Shirk, J. Phys. D 42, 175304 (2009). 